\title{
Kreuzweh: Frühe Physiotherapie nützt wenig
}

\author{
Eine frühe Physiotherapie bei Patienten mit akuten lumbalen Rückenschmerzen führt nur \\ zu einer marginalen Verbesserung der Behinderung und der Schmerzintensität.
}

_ Rückenschmerzen sind für etwa 3-5\% aller Arztbesuche verantwortlich, die meisten davon beim Hausarzt. Bei chronischen Formen ist der Nutzen von Krankengymnastik und Physiotherapie belegt. Für die Akutsituation gibt es bisher nur wenige Daten.

Für eine randomisierte klinische Studie wurden 220 Teilnehmer zwischen 18 und 60 Jahren mit akuten Rückenschmerzen in den letzten 16 Tagen rekrutiert. Auf dem Oswestry Disability Index (ODI) erreichten sie einen Wert von mindestens 20, im Mittel wurde ein Wert von 40 erreicht. Die mittlere Schmerzintensität auf einer Skala von 0 bis 10 wurde mit 5 angegeben. Patienten mit radikulären Schmerzen wurden ausgeschlossen. Der Frauenanteil lag bei 50\%. Zwei Drittel der Teilnehmer nah- men nicht-steroidale Antirheumatika und ein Drittel Opioide ein.

Alle Patienten erhielten eine Verhaltensschulung, 108 von ihnen zusätzlich vier Sitzungen Physiotherapie, in der Regel 72 Stunden nach dem ersten Arztkontakt. Dabei kamen u. a. Manualtherapie, Bewegungs- und Kraftübungen zum Einsatz. Der primäre Outcome war die Veränderung auf dem ODI nach vier Wochen und nach einem Jahr. Außerdem wurden Schmerzintensität, Lebensqualität und Inanspruchnahme des Gesundheitssystems untersucht.

Nach drei Monaten fand sich ein signifikanter Unterschied zugunsten der Physiotherapie für die allgemeine Behinderung und nach vier Wochen für den ODI-Score. Unterschiede bezüglich der Schmerzintensität ließen sich nicht nachweisen. Auch waren in keiner der Gruppen Arztbesuche häufiger.

- Fritz JM, Magel JS, McFadden M et al. Early physical therapy vs usual care in patients with recent-onset low back pain: $A$ randomized clinical trial. JAMA. 2015;314:1459-67

\section{KOMMENTAR}

Die Studie zeigt, dass bei akuten Rückenschmerzen eine frühe Physiotherapie Beschwerden und Behinderungen nach vier Wochen leicht bessert. Alle Patienten wurden allerdings auch medikamentös behandelt. Für den Alltag bedeutet dies, dass zunächst der Verlauf unter NSAR-Therapie abgewartet werden kann. Bei starken Schmerzen sind retardierte Opioide angezeigt. Bestehen die Schmerzen länger als 10 bis 14 Tage, sollten dann allerdings Physiotherapie und Krankengymnastik zum Einsatz kommen.

Prof. Dr. med. H.-C. Diener

\section{Madelung-Krankheit: Überzogen von Fetteinlagerungen}
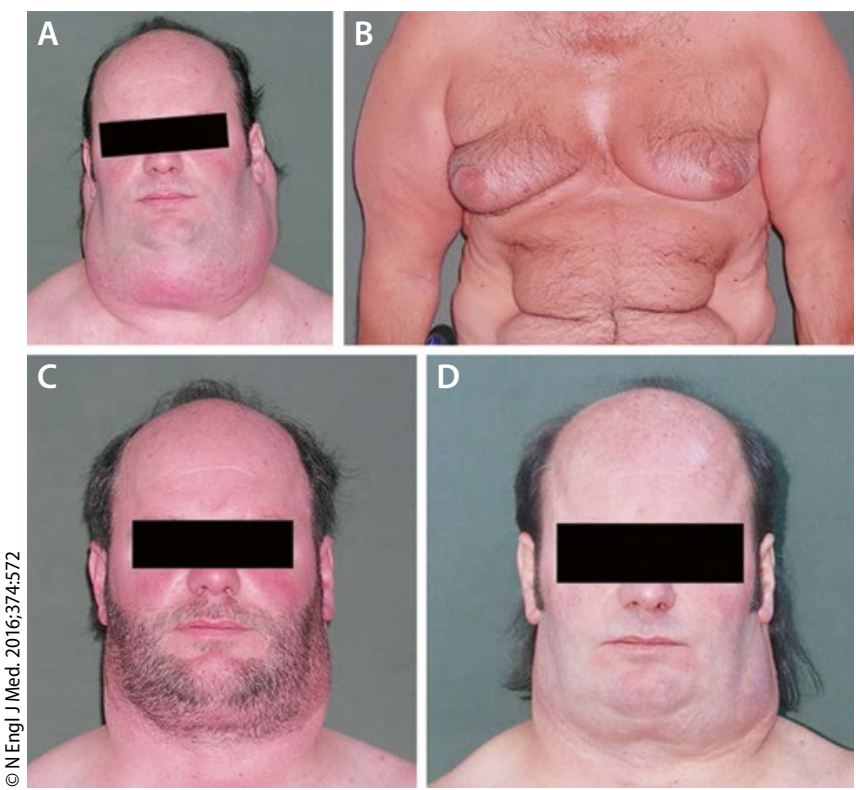

Fetteinlagerungen an Kopf und Oberkörper (A, B), chirurgisches Ergebnis nach einem Jahr (C), Rezidive nach drei Jahren (D).
Ein 48-jähriger Mann stellte sich wegen Schluckbeschwerden und eingeschränkter Beweglichkeit im Nacken in der Ambulanz vor. Aufgrund des typischen Aspekts stellte man die Diagnose einer Madelung-Krankheit, die auch unter dem Begriff der benignen symmetrischen Lipomatose oder dem Launois-Bensaude-Syndrom bekannt ist. Die Madelung-Erkrankung ist charakterisiert durch multiple, symmetrische und entstellende abnorme Fetteinlagerungen im Bereich von Kopf, Hals, Stamm und den Nervenwurzeln der oberen und unteren Extremitäten (Abb. A und B). Die Patienten haben oft weitere Erkrankungen wie Leberschäden, Diabetes, Polyneuropathie, Gynäkomastie, Hyperurikämie und Dyslipidämie. Die Erkrankung ist mit Alkoholmissbrauch assoziiert, es gibt aber auch familiäre Formen mit mütterlichem Übertragungsweg. Ätiologie und Pathogenese sind weitgehend unbekannt, es bestehen allerdings Hinweise auf eine mitochondriale Dysfunktion mit reduzierter Aktivität des Enzyms Cytochrom-c-Oxidase. Die Therapieoptionen erschöpfen sich in der Entfernung des Fettgewebes.

Der Patient wurde operiert und war mit dem funktionellen und ästhetischen Ergebnis ein Jahr danach weitgehend zufrieden (Abb. C). Rezidive sind häufig, so auch bei diesem Patienten drei Jahre nach der ersten chirurgischen Behandlung (Abb. D).

Prof. Dr. med. H. S. FüeßI

- Nisi G, Sisti A (asisti6@gmail.com). Madelung's disease. NEngl J Med. 2016;374:572 